\title{
Medium Range Forecast of Maximum Temperature of Coimbatore using ANN
}

\author{
D. Abinayarajam $^{1 *}$, S. G. Patil ${ }^{1}$, Ga. Dheebhakaran ${ }^{2}$ and S. P. Ramanathan ${ }^{2}$ \\ ${ }^{1}$ Agricultural Statistics, ${ }^{2}$ Agro Climate Research Centre, Tamil Nadu Agricultural University, \\ Coimbatore, India \\ *Corresponding author
}

A B S T R A C T

Keywords

ANN, NNAR,

Maximum

temperature, RMSE

Article Info

Accepted:

18 December 2020

Available Online:

10 January 2021
Climate is the most important dominant factor influencing the plant growth for particular region. Artificial neural network is a nonlinear modelling used for efficient forecast of weather parameter. Daily data on maximum temperature were collected from Agro climatic Research Centre, TNAU over the period of 1991 - 2017.Analysis has been carried out using the R software 3.4.1. Datasets were split into training and test datasets. The training data were used as input. It has been observed that, NNAR model $(6,1,5)_{[365]}$ was predict the maximum temperature with greater accuracy.

\section{Introduction}

In agricultural development, the weather plays an important role. It has a profound impact on a crop's growth, development, and yields, pest and disease incidence, irrigation, and fertilizer requirements. The weekly weather favours outbreak of pest and disease which hamper the crop yield. Predicting weather parameter help in such activity so has to get better output (returns) from agriculture. Forecasting of weather condition up to three days is known as shortrange forecasting. Forecasting up to seven days is known as medium-range forecasting. Forecasting up to thirty days is known as longrange forecasting.
Toth et al., (2000) studied the comparison of short-term rainfall prediction models for realtime flood forecasting. Zhang (2003)using a hybrid ARIMA and neural network model to forecast time series data. Bilgili et al., (2007) studied the application of an artificial neural networks for the wind speed prediction of target stations using the reference stations data. Pandey et al., (2008) compared the neural network and fuzzy model to forecast wheat production in India. Gasse et al., (2014)applied the hybrid algorithm for Bayesian network structure learning. In this study, an attempt has been made to predict the maximum temperature of Coimbatore using ANN. 


\section{Materials and Methods}

\section{Data collection}

The secondary data on maximum temperature of Coimbatore were collected from agro climatic research centre of Tamil Nadu agricultural university, Coimbatore. Daily data were taken from the periods of $1^{\text {st }}$ January 1991 to $10^{\text {th }}$ December 2017 with 9841 observations.

\section{Artificial neural network}

An Artificial Neural Network (ANN) is a computational model based on the structure and functions of biological neural networks. ANNs are considered nonlinear statistical data modelling tools where the complex relationships between inputs and outputs are modelled or patterns are found.

Multiplication, summation, and activation are the three simple sets of rules in ANN. The input is multiplied by its appropriate weights. The weights represent the interconnection between the neuron in the neural network. The weighted inputs are all summed up inside the computing unit and bias. The sum is passed through a nonlinear function known as an activation function or transfer function. The activation function is usually having a sigmoid shape. The output is

$\hat{Y}=f^{\left\{\sum_{j}^{q} w_{k j} f\left(\sum_{i}^{p} w_{i j} x_{i}+b_{j}\right)+b_{k}\right\}}$

Where $f$ is an activation function, $x_{\tilde{i}}$ is the input vector and ' $b$ ' is the bias, ' $p$ ' is the number of neurons in the input layer, ' $\mathrm{q}$ ' is the number of neurons in the output layer, ' $w$ ' is the weight and $\mathrm{i}, \mathrm{j}, \mathrm{k}$ are the neurons of the input, middle and output respectively.

\section{Accuracy measures}

The difference between the original value and the predicted value is known as residuals. Assume $y_{i}$ denote the $i^{\text {th }}$ observation of $y$ and $\hat{y}_{i}$ is predicted value of $\mathrm{y}_{\mathrm{i}}$, then residuals are

$\mathbf{e}_{\mathrm{i}}=\left(\mathrm{y}_{\mathrm{i}}-\widehat{\mathrm{y}}_{\mathrm{i}}\right)$

Scale-dependent errors denote that data and the residuals are on the same scale. The most commonly used scale-dependent errors is Root mean squared error $(\mathrm{RMSE})=\sqrt{\text { mean }\left(\mathrm{e}_{\mathrm{t}}^{2}\right)}$. The model which have a minimum value of error is considered the best model.

\section{Results and Discussion}

Neural Network Auto regression was fitted to maximum temperature data with help of $\mathrm{R}$ software. Daily weather data on maximum temperature with 9841 observations was spilt into training datasets and test data sets. The last 10 days' data points consider testing data sets, to validate the medium-range forecast. Training datasets were normalized and 20 repeats were used in neural network auto regression. In NNAR $(\mathrm{p}, \mathrm{P}, \mathrm{k})$ where $\mathrm{p}$ indicates the lagged value of non-seasonal time series $\operatorname{AR}(\mathrm{p}), \mathrm{p}$ is the lagged inputs for seasonal time series $\mathrm{AR}(\mathrm{P})$. and $\mathrm{k}$ indicates the nodes in the hidden layer. NNAR model $(6,1,5){ }_{[365]}$ for daily data on maximum temperature was best fitted with greater accuracy. Root mean square value is used for model accuracy. This NNAR model $(6,1,5)$ [365] has RMSE value of 2.35 .

In conclusion the neural network Auto regression model was fitted to weather data. NNAR model $(6,1,5)$ [365] Was predict the maximum temperature of Coimbatore with greater accuracy. 
Table.1 Actual vs fitted values of maximum temperature Coimbatore

\begin{tabular}{|l|c|c|}
\hline Days & Actual & ANN \\
\hline D1 & 24.50 & 29.83 \\
\hline D2 & 26.50 & 29.05 \\
\hline D3 & 25.20 & 28.43 \\
\hline D4 & 29.00 & 29.04 \\
\hline D5 & 30.50 & 29.53 \\
\hline D6 & 30.50 & 29.84 \\
\hline D7 & 31.00 & 30.04 \\
\hline D8 & 31.50 & 30.06 \\
\hline D9 & 32.00 & 30.21 \\
\hline D10 & 32.00 & 30.39 \\
\hline
\end{tabular}

Fig.1 Working principle of ANN

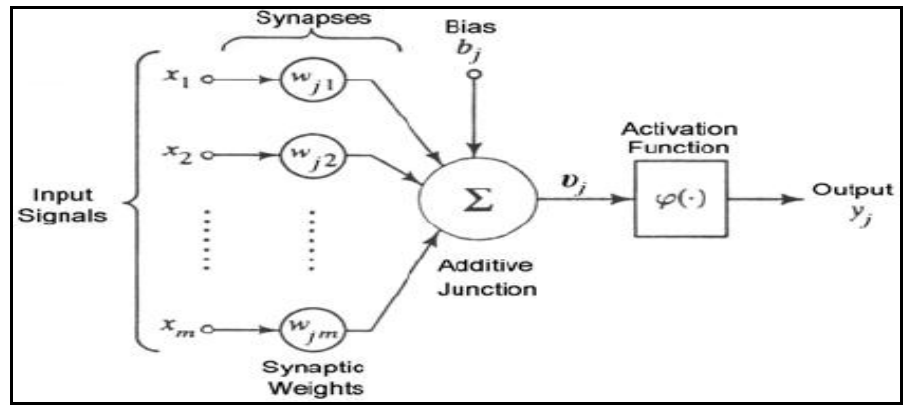

Fig.2 Actual vs fitted plot of maximum temperature

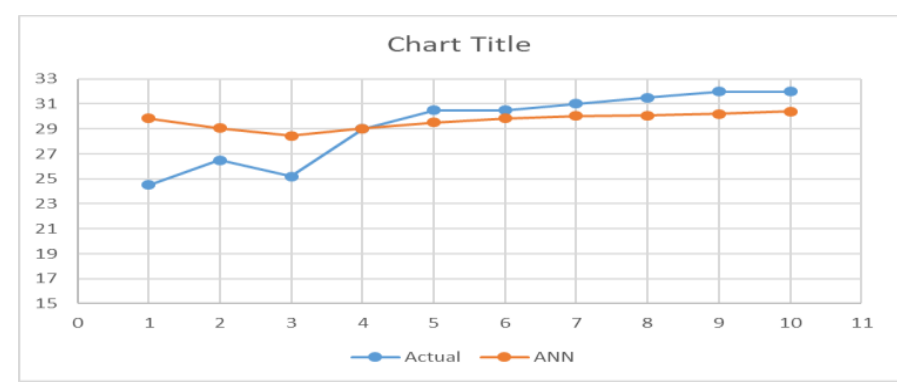

\section{References}

Bilgili, M., B. Sahin, and A. Yasar. 2007. Application of artificial neural networks for the wind speed prediction of target station using reference stations data. Renewable Energy 32 (14):2350-2360.

Gasse, M., A. Aussem, and H. Elghazel. 2014. "A hybrid algorithm for Bayesian network structure learning with application to multi-label learning." Expert Systems with Applications 41 (15):6755-6772.

Litta, A., S. Mary Idicula, and U. Mohanty. 2013. Artificial neural network model in prediction of meteorological parameters during premonsoon thunderstorms. International Journal of atmospheric 
sciences 2013.

Pandey, A.K., A. Sinha, and V. Srivastava. 2008. A Comparative Study of NeuralNetwork \& Fuzzy Time Series Forecasting Techniques-Case Study: Wheat Production Forecasting. International Journal of computer science and network security 8 (9):382387.

Singh, A., and G. Mishra. 2015. Application of Box-Jenkins method and Artificial Neural Network procedure for time series forecasting of prices. Statistics in Transition new series 1 (16):83-96.

Toth, E., A. Brath, and A. Montanari. 2000. Comparison of short-term rainfall prediction models for real-time flood forecasting. Journal of hydrology 239 (1-4):132-147.

Zhang, G.P. 2003. Time series forecasting using a hybrid ARIMA and neural network model. Neurocomputing 50:159-175.

\section{How to cite this article:}

Abinayarajam, D., S. G. Patil, Ga. Dheebhakaran and Ramanathan, S. P. 2021. Medium Range Forecast of Maximum Temperature of Coimbatore using ANN. Int.J.Curr.Microbiol.App.Sci. 10(01): 3015-3018. doi: https://doi.org/10.20546/ijcmas.2021.1001.349 